\title{
Expression of the FAM5C in tongue squamous cell carcinoma
}

\author{
TSUKASA KUROIWA, NOBUHARU YAMAMOTO, TAKESHI ONDA and TAKAHIKO SHIBAHARA \\ Department of Oral and Maxillo-Facial Surgery, Tokyo Dental College, 1-2-2 Masago, Mihama-ku, Chiba 261-8502, Japan
}

Received May 19, 2009; Accepted July 10, 2009

DOI: 10.3892/or_00000528

\begin{abstract}
The purpose of this study was to perform a wholegenome analysis of loss of heterozygosity $(\mathrm{LOH})$ in tongue squamous cell carcinoma (SCC) using the Affymetrix 10K SNP Mapping Array. In the gene which had been identified by whole-genome analysis of $\mathrm{LOH}$, we analyzed allelic imbalance to identify the role of the gene. We applied wholegenome analysis of $\mathrm{LOH}$ in the specimens from the 5 cases of tongue SCC using this array. In the chromosomal region which had been identified by whole-genome analysis of $\mathrm{LOH}$, we reconfirmed the existence of $\mathrm{LOH}$ in 30 cases using microsatellite markers. The expression levels of the mRNA in the region were examined in 15 cases and in 5 tongue SCC-derived cell lines by real-time quantitative RTPCR analysis. LOH was observed in all of the 5 cases in the 1q31.1 region. Only 3 microsatellite markers (D1S1189, D1S2151, and D1S2595) existed in the 1q31.1 region. A high frequency of LOH was found at the D1S1189 locus in 18/30 (60\%), D1S2151 locus in 16/30 (53\%) and D1S2595 locus in 21/30 (70\%). Only the Family with sequence similarity 5, member $\mathrm{C}$ (FAM5C) gene was located in the 1q31.1 region. There was statistically significant difference in the FAM5C mRNA expression levels between tongue SCC and normal tissues. All tongue SCC-derived cell lines decreased FAM5C mRNA expression compared with normal oral keratinocytes (NOKs). We conclude that FAM5C may be a novel tumor suppressor gene (TSG) in tongue SCC.
\end{abstract}

\section{Introduction}

Inactivation of tumor suppressor genes (TSGs) and activation of oncogenes have been considered to play important roles in the multi-step process of human carcinogenesis. Chromosomal loss of heterozygosity ( $\mathrm{LOH})$ is a common mechanism for the inactivation of TSGs in human carcinoma. $\mathrm{LOH}$ patterns can be generated through allelotyping using polymorphic microsatellite markers (1).

Correspondence to: Dr Takahiko Shibahara, Department of Oral and Maxillo-Facial Surgery, Tokyo Dental College, 1-2-2 Masago, Mihama-ku, Chiba 261-8502, Japan

E-mail: sibahara@tdc.ac.jp

Key words: FAM5C, tongue squamous cell carcinoma, loss of heterozygosity, copy number abnormality, tumor suppressor gene
Several studies demonstrated that multiple genetic alterations were involved in the carcinogenetic process in head and neck squamous cell carcinoma (SCC). Among these alterations, frequent $\mathrm{LOH}$ at many chromosome loci was the most striking, indicating that multiple TSGs played important roles in head and neck SCC carcinogenesis (2).

In our laboratory, Yamamoto et al analyzed chromosomes $2 q, 3 p$ and $21 q$ for LOH in 40 primary oral SCCs using 30 markers and constructed a deletion map for these chromosome arms. LOH were observed at 2 loci in chromosome 2q, 3 loci in chromosome $3 p$, and 4 loci in chromosome 21q. Our studies suggested that allelic deletions of the $2 q, 3 p$, and $21 q$ loci played a role in oral SCC progression (2). However, because of the limited number of available microsatellite markers, the rather tedious and labor-intensive procedure, and the requirement for large amounts of DNA, only a modest number of microsatellite markers could be screened (3).

High-density whole genome allelotyping cannot be performed; however, single nucleotide polymorphisms (SNPs) offer many advantages for genetic analysis, including their prevalence in the genome and ease of assay. The unique advantage of SNPs for LOH analyses are that, unlike microsatellites, they are not susceptible to the repeat expansion so often observed in cancer and thus provide higher-fidelity markers for tracking the fate of chromosome segments. Analysis using the Affymetrix ${ }^{\circledR} 10 \mathrm{~K}$ SNP Mapping Array is, therefore, a logical approach for genome-wide allelic imbalance profiling and an efficient method for detecting genome-wide cancer LOH (3).

Several studies have successfully utilized this array for identifying consistent LOH regions in breast cancer (4-8), bladder cancer $(9,10)$, prostate cancer $(11,12)$, osteosarcoma $(13)$, lung cancer $(1,14)$ and oral SCC-derived cell lines $(3,15)$. However, only a limited number of studies have utilized this assay for identifying consistent $\mathrm{LOH}$ regions in human oral SCCs.

In this study, we analyzed existence of $\mathrm{LOH}$ in human tongue SCCs using this array. Then, in the chromosomal region identified by the genome-wide $\mathrm{LOH}$ detection, we reconfirmed the existence of $\mathrm{LOH}$ using microsatellite markers. The mRNA expression levels of the gene harbored in this region were examined to identify the role of the gene.

\section{Materials and methods}

Cells. The five human tongue SCC-derived cell lines used in this study were SCC-4, HSC-3, HSC-4, OSC-19, and OSC-20 (Human Science Research Resources Bank, Osaka, Japan). All 
Table I. Summary of clinicopathological features in 30 tongue SCCs.

\begin{tabular}{|c|c|c|c|c|c|c|c|c|c|c|}
\hline Case & Gender & Age & $\mathrm{T}$ & $\mathrm{N}$ & Stage & $\mathrm{pN}$ & Differentiation & Prognosis & Progress & Final \\
\hline 1 & M & 41 & 2 & $2 b$ & IV & - & Well & Well & Well & Alive \\
\hline 2 & M & 58 & 1 & 1 & III & + & Well & Well & Well & Alive \\
\hline 3 & M & 63 & 1 & 0 & I & - & Well & Well & Well & Alive \\
\hline 4 & M & 33 & 2 & 1 & III & + & Well & Well & Well & Alive \\
\hline 5 & $\mathrm{~F}$ & 60 & 4 & $2 b$ & IV & + & Well & Well & Well & Alive \\
\hline 6 & $\mathrm{~F}$ & 63 & 2 & 0 & II & - & Well & Well & Well & Alive \\
\hline 7 & M & 42 & 1 & 0 & I & - & Poor & Well & Well & Alive \\
\hline 8 & M & 68 & 1 & 1 & III & - & Well & Poor & Death & Died \\
\hline 9 & $\mathrm{M}$ & 56 & 3 & $2 c$ & IV & + & Well & Well & Well & Alive \\
\hline 10 & $\mathrm{M}$ & 38 & 1 & 0 & I & + & Poor & Poor & Meta & Alive \\
\hline 11 & $\mathrm{M}$ & 53 & 2 & $2 b$ & IV & + & Well & Well & Well & Alive \\
\hline 12 & $\mathrm{M}$ & 47 & 2 & $2 b$ & IV & + & Well & Well & Well & Alive \\
\hline 13 & $\mathrm{M}$ & 52 & 1 & 0 & I & - & Well & Well & Well & Alive \\
\hline 14 & $\mathrm{M}$ & 44 & 2 & 0 & II & - & Well & Well & Well & Alive \\
\hline 15 & $\mathrm{~F}$ & 54 & 2 & $2 c$ & IV & + & Well & Well & Well & Alive \\
\hline 16 & $\mathrm{~F}$ & 58 & 1 & 0 & I & - & Well & Well & Well & Alive \\
\hline 17 & $\mathrm{M}$ & 67 & 1 & 0 & I & - & Well & Well & Well & Alive \\
\hline 18 & $\mathrm{~F}$ & 63 & 1 & 0 & I & - & Well & Well & Well & Alive \\
\hline 19 & $\mathrm{M}$ & 52 & 1 & 0 & I & - & Well & Well & Well & Alive \\
\hline 20 & $\mathrm{M}$ & 57 & 2 & $2 b$ & IV & - & Well & Well & Well & Alive \\
\hline 21 & $\mathrm{M}$ & 43 & 1 & 0 & I & - & Well & Well & Well & Alive \\
\hline 22 & $\mathrm{M}$ & 58 & 2 & $2 b$ & IV & + & Well & Well & Well & Alive \\
\hline 23 & $\mathrm{M}$ & 66 & 2 & $2 b$ & IV & - & Well & Well & Well & Alive \\
\hline 24 & $\mathrm{M}$ & 64 & 4 & $2 c$ & IV & + & Well & Well & Well & Alive \\
\hline 25 & M & 62 & 2 & 0 & II & + & Well & Poor & Meta & Alive \\
\hline 26 & $\mathrm{M}$ & 61 & 2 & 0 & II & - & Well & Well & Well & Alive \\
\hline 27 & M & 75 & 4 & $2 b$ & IV & + & Poor & Poor & Meta & Died \\
\hline 28 & $\mathrm{M}$ & 85 & 2 & 0 & II & - & Well & Well & Well & Alive \\
\hline 29 & M & 55 & 2 & $2 c$ & IV & + & Well & Well & Well & Alive \\
\hline 30 & $\mathrm{M}$ & 48 & 1 & 0 & I & - & Well & Well & Well & Alive \\
\hline
\end{tabular}

M, Male; F, Female; pN, pathological Node; Well, well differentiated; Mod, moderately differentiated; Poor, poorly differentiated; Meta, metastasis.

cell lines were maintained at $37^{\circ} \mathrm{C}$ (humidified atmosphere $5 \% \mathrm{CO}_{2} / 95 \%$ air) on $150 \times 20 \mathrm{~mm}$ tissue culture dishes (Nunc, Roskilde, Denmark) and cultured in Dulbecco's modified Eagle's medium F-12 HAM (Sigma, St. Louis, MO, USA) with $10 \%$ fetal bovine serum (Sigma) plus $50 \mathrm{U} / \mathrm{ml}$ penicillin and streptomycin (16).

Two normal oral keratinocyte (NOK) strains from patients who had undergone dental surgery served as the controls, and the two patients provided written informed consent before the start of the study. The normal oral specimens were washed in Dulbecco's phosphate-buffered saline (PBS) (Sigma) and then placed overnight in $0.25 \%$ trypsin-EDTA solution (Sigma) at $4^{\circ} \mathrm{C}$. After the epithelial tissue was separated from the connective tissue, it was disaggregated by incubation in $0.25 \%$ trypsin-EDTA solution for $15 \mathrm{~min}$ with gentle pipetting at $37^{\circ} \mathrm{C}$. Isolated epithelial cells then were seeded into Collagen I Cellware 60-mm dish biocoat cell environments (BectonDickinson Labware,
Bedford, MA, USA) and cultured in Keratinocyte Basal Medium-2 (Cambrex, Walkersville, MD, USA) with $0.4 \%$ bovine pituitary extract, $0.1 \%$ human epidermal growth factor, $0.1 \%$ insulin, $0.1 \%$ hydrocortisone, $0.1 \%$ transferrin, $0.1 \%$ epinephrine, and $0.1 \%$ GA-1000 (Cambrex) (17).

Tissue samples. Sample tissues were taken from 30 patients with oral tongue SCC who attended the Tokyo Dental College of Chiba Hospital, during the 3 years between 2005 and 2007 (Table I). Informed consent was obtained from all patients, and our protocol was reviewed and approved by the institutional review board of Tokyo Dental College. Thirty primary tumors and 30 normal tissues corresponding to these primary tumors comprised the tissue samples. They were collected either at the time of surgical resection or at the time of biopsy.

The resected tissues were divided into two segments; one was frozen immediately after careful removal from the surrounding normal tissues and stored at $-80^{\circ} \mathrm{C}$ until DNA 
Table II. Microsatellite markers used in our LOH study.

\begin{tabular}{lccc}
\hline Markers & Locations & $\begin{array}{c}\text { Size of PCR } \\
\text { Products (bp) }\end{array}$ & Sequence of primers \\
\hline D1S1189 & $1 \mathrm{q} 31.1$ & 341 & 5'-CTGAACTAACACGGAGAAAC-3' \\
5'-GTAGACTGTTAAAAGAAGAGC-3' & 5'-CTGTATAAAGAGCGCTGTGGG-3' \\
D1S2151 & $1 \mathrm{q} 31.1$ & 260 & 5'-GGATGGGGCAGTGAGAAG-3' \\
& $1 \mathrm{q} 31.1$ & & 5'-GGGCGTTCCAATACTTAGAGG-3' \\
& & 102 & 5'-AGGACACCACAAGTTCCAGG-3' \\
\hline
\end{tabular}

and RNA extraction, and the other one was fixed in $10 \%$ formalin for pathologic diagnosis. Histopathologic diagnosis was performed according to the International Classification of Tumors. Clinicopathologic staging was determined using the UICC TNM staging system.

DNA extraction. All patients had histologically confirmed tongue SCC, and the tumor samples for DNA extraction were checked to ensure that they consisted of $>80 \%$ tumor. All tumor samples and most normal tissue samples were processed from freshly frozen specimens that had been preserved in liquid nitrogen immediately after resection. First, the tissues were powdered with liquid nitrogen. After they had been collected by single centrifugation, genomic DNAs were then added to $1000 \mu 1$ of TNES buffer $(10 \mathrm{mM}$ Tris- $\mathrm{HCl}$ (pH 8.0), $150 \mathrm{mM} \mathrm{NaCl}, 10 \mathrm{mM}$ EDTA, 0.1\% SDS) and $30 \mu \mathrm{l}$ of Proteinase $\mathrm{K}(100 \mu \mathrm{g} / \mathrm{ml})$, stirred, digested, and incubated in a water-bath overnight at $50^{\circ} \mathrm{C}$. After the DNAs had been extracted with phenol-chloroform and refined, they were washed and precipitated with ethanol. The concentrations of the extracted DNAs were estimated by a spectrophotometric method, and the DNAs were kept frozen at $-80^{\circ} \mathrm{C}$. From each DNA sample, $50 \mathrm{ng} / \mu \mathrm{l}$ was used as the template for the polymerase chain reaction (PCR) amplification procedure.

For the analysis of DNA mapping array, normal cells were prepared from peripheral blood samples as a control. The normal blood samples were analyzed with the Dr.GenTLE ${ }^{\circledR}$ (Takara Bio Inc., Shiga, Japan) gene trapping kit (18).

Analysis of DNA mapping array using Affymetrix 10K SNP Mapping Array. Five cases of tongue SCC were subjected to DNA mapping array analysis of all chromosomes using the Affymetrix 10K SNP Mapping Array to determine the presence of $\mathrm{LOH}$.

DNA labeling, hybridization, washing and staining of the Affymetrix 10K SNP Mapping Array were performed according to the standard Single Primer GeneChip Mapping $10 \mathrm{~K}$ Assay protocol (Affymetrix). The array was scanned with a GeneChip Scanner 3000, and the scanned array images were processed with GeneChip Operating software (GCOS). The genotype calls and intensities of the SNP probes were generated by GeneChip DNA Analysis software (GDAS). Individual SNP copy numbers and chromosomal regions with gains or losses were evaluated with the Affymetrix GeneChip chromosome copy number tool 2.0 (19).
Analysis of $\mathrm{LOH}$ using microsatellite markers. In the region which had been identified by whole-genome analysis of $\mathrm{LOH}$, we reconfirmed the existence of $\mathrm{LOH}$ in 30 cases using microsatellite markers. Only 3 microsatellite markers (D1S1189, D1S2151, and D1S2595) existed in the 1q31.1 region. All primers were obtained from Sigma-Aldrich Japan K.K. (Hokkaido, Japan) (Table II). LOH in the tumor DNA samples was assessed by scanning densitometry and analyzed with NIH Image software (version 1.62, http://rsb.info.nih.gov/ nih-image/). The intensities of the signals from the tumor DNA were compared with those of normal control DNA. The criterion for the presence of $\mathrm{LOH}$ was a reduction in signal intensity of $>50 \%$. Commonly deleted regions were defined by considering the loci most frequently showing $\mathrm{LOH}$, together with multiple interstitial deletions (18).

$m R N A$ expression analysis. Only the Family with sequence similarity 5, member $\mathrm{C}$ (FAM5C) gene was located in the 1q31.1 region (NCBI Entrez Gene: http://www.ncbi.nlm.nih. gov/entrez). Fifteen cases of tongue SCC were examined the expression levels of FAM5C mRNA. The expression levels of FAM5C mRNA were examined in the tumors, and paired normal oral tissues from the 15 cases with tongue SCC, five tongue SCC-derived cell lines, and two NOKs. We selected 15 cases who could be extracted total RNA. Control reactions were prepared in parallel without reverse transcriptase. Before cDNA synthesis, residual genomic DNA was removed from the total RNA by DNase I treatment (DNA-free; Ambion, Austin, TX, USA). The primer sequences used for analysis of FAM5C mRNA expression were forward: 5'-ACCTGAG AAGTCGCATCAAG-3' and reverse: 5'-CCAGGTGGG GAGAGTTTATT-3'. The sequence of specific primers was checked before use to avoid amplification of genomic DNA or pseudogenes using the Primer3 program (http://wwwgenome.wi.mit.edu/cgibin/primer/primer3_www.cgi). The amplified products were analyzed by $3 \%$ agarose gel electrophoresis to ascertain the size and purity of the products. To confirm the identity of the PCR products, they were cloned into a pCR 2.1vector (Invitrogen Japan, K.K., Tokyo, Japan) and sequenced as described previously (20).

Real-time quantitative RT-PCR was performed by a single method using a LightCycler FastStart DNA Master SYBR Green I kit (Roche Diagnostics GmbH, Mannheim, Germany). To prepare the standard curve, $1.5 \mu \mathrm{g}$ of total RNA from normal oral tissue was reverse transcribed with Superscript 


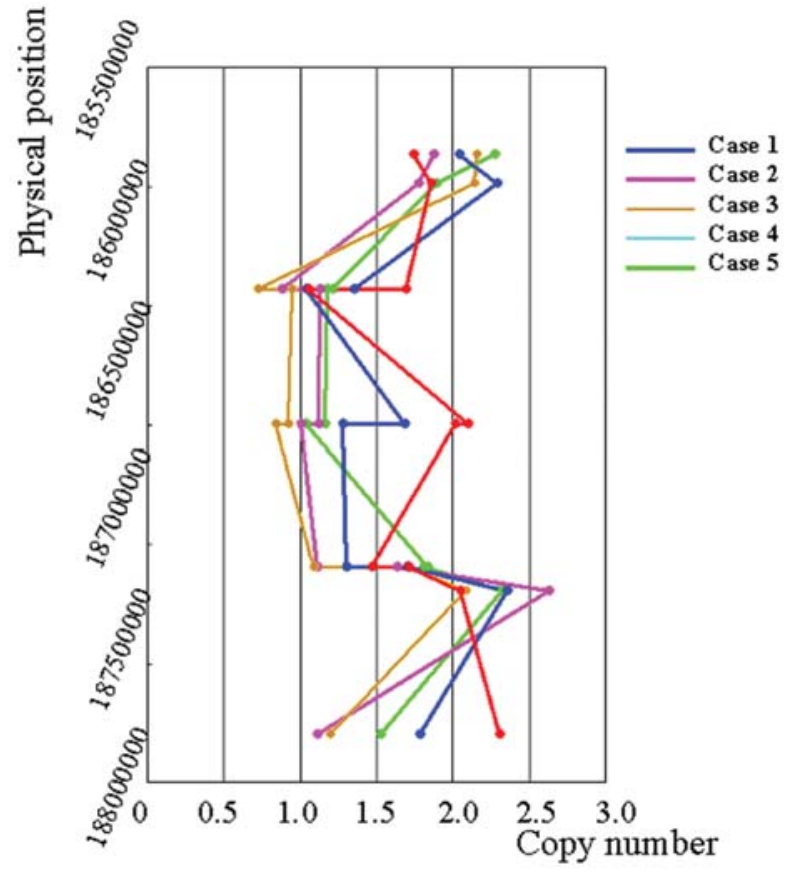

Figure 1. Analysis of copy number abnormality (CNA) on 1q31.1 region. Physical positions of each SNP probe are shown at the vertical axis. The copy number of each SNP probe is shown at the horizontal axis. In the region of 1q31.1, genome copy numbers from 0.8 to 1.2 were confirmed from continual SNP probes in all of the 5 cases.

reverse transcriptase (Life Technologies, Grand Island, NY, USA) and an oligo-d (T) primer, after which serial dilutions were made corresponding to the cDNA transcribed from 300 , 30, 3.0, and 0.3 ng of total RNA. The PCR reactions using the LightCycler (Roche Diagnostics) apparatus were carried out in a final volume of $20 \mu 1$ of the reaction mixture consisting of $2 \mu 1$ of FastStart DNA Master SYBR Green I mix (Roche), and $3 \mathrm{mM} \mathrm{MgCl}_{2}$, and $0.2 \mu 1$ of the primers, according to the manufacturer's instructions. The reaction mixture was loaded into glass capillary tubes and submitted to an initial denaturation at $95^{\circ} \mathrm{C}$ for $10 \mathrm{~min}$, followed by 45 rounds of amplification at $95^{\circ} \mathrm{C}(10 \mathrm{sec})$ for denaturation, $56^{\circ} \mathrm{C}(10 \mathrm{sec})$ for annealing, and $72^{\circ} \mathrm{C}$ for extension, with a temperature slope of $20^{\circ} \mathrm{C}$, performed in the LightCycler. The amount of transcript from the FAM5C gene was estimated from the respective standard curves and normalized to the amount of glyceraldehyde-3-phosphate dehydrogenase (GAPDH) transcript determined in corresponding samples (16).

Clinicopathologic findings and statistical analysis. The patients, 25 males and 5 females, had an average age of 55.5 years for the males (range: 33-80 years) and 59.6 years for the females (range: 54-63 years). The T classifications, which indicate the sizes of the primary clinical tumors, were: 12 patients with T1, 14 with T2, 1 with $\mathrm{T} 3$, and 3 with T4. The classifications by TNM stage were: 10 patients with Stage I, 5 with Stage II, 3 with Stage III, and 12 with Stage IV. Thirteen among the 30 patients had histopathologically confirmed cervical lymph node metastasis (LNM) at the time of diagnosis or during the 8-month follow-up period (LNM present). The grades of histological differentiation were: 27 well differentiated tumors and 3 poorly differentiated ones. The prognoses obtained were: 26 favorable prognoses and 4 poor prognoses. The Fisher's exact test was performed to evaluate the significance of correlations between $\mathrm{LOH}$ and clinicopathological findings. The accepted level of significance was $\mathrm{P}<0.05$.

\section{Results}

Analysis of DNA mapping array using Affymetrix 10K SNP Mapping Array. We applied whole-genome analysis of LOH in the specimens from the 5 cases of tongue SCC using

$\underline{\text { Deletion mapping }}$

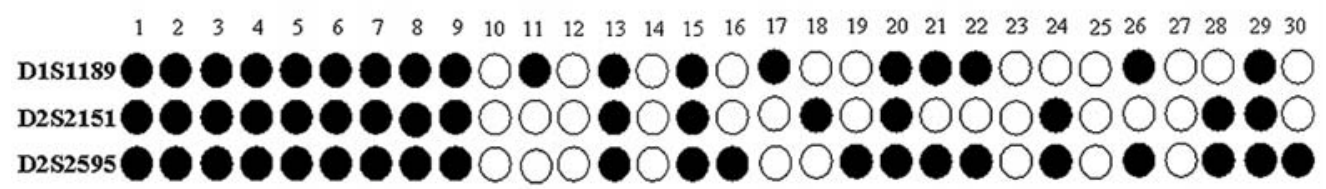

$\mathrm{LOH}:$ loss of heterozygosity

ROH : retention of heterozygosity

Illustration of microsatellite polymorphism analysis

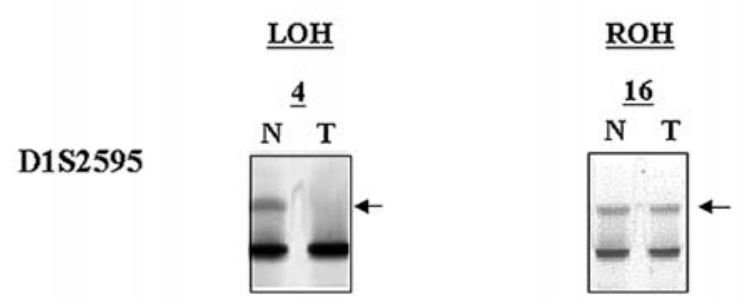

Figure 2. Deletion mapping in the 1q31.1 region in 30 tongue SCCs and illustration of microsatellite polymorphism analysis. Deletion mapping: Case numbers are shown at the top and locus symbols on the left. Illustration of microsatellite polymorphism analysis: Case numbers are shown at the top, and locus symbols on the left. Paired normal (N) and tumor (T) cases for patients 4 and 16 demonstrating loss of heterozygosity (LOH) and retention of heterozygosity $(\mathrm{ROH})$. 


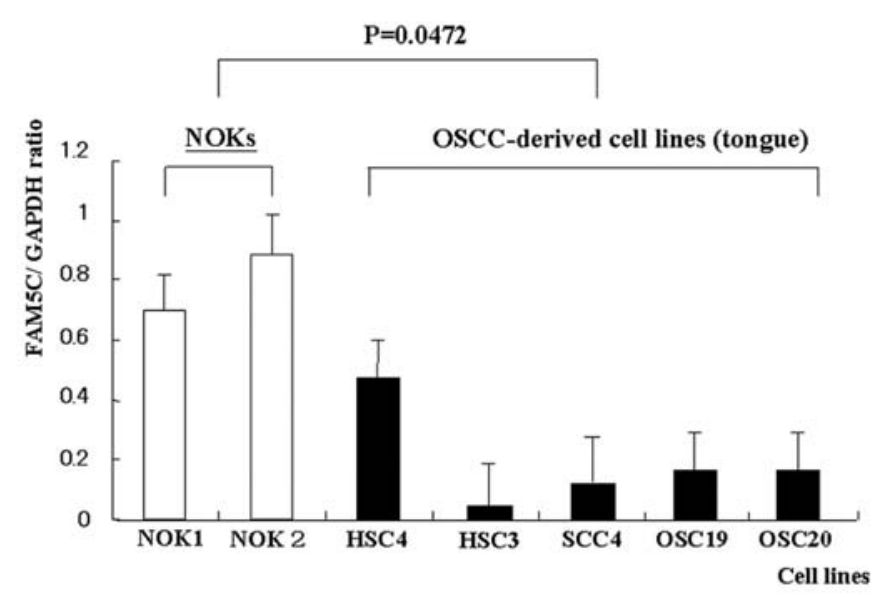

Figure 3. Quantification of mRNA levels in tongue SCC-derived cell lines by real-time RT-PCR analysis. All tongue SCC-derived cell lines decreased FAM5C mRNA expression compared with NOKs. There was statistically significant difference in the FAM5C mRNA expression levels between tongue SCC-derived cell lines and the NOKs $(\mathrm{P}=0.0472$, Mann-Whitney U test).

Affymetrix 10K SNP Mapping Array. In this study, we observed the loss of copy number in copy number abnormality (CNA). At the analysis using this array, a CNA (one copy) suggested LOH (15). A CNA (one copy) was observed in the regions of 1q31.1, 3q13.13, 6p12.3, 9p13.2, 9q33.1, 10p11.21, and $20 \mathrm{p} 11.21$. In the region of $1 \mathrm{q} 31.1$, genome copy numbers from 0.8 to 1.2 were confirmed using continual SNP probes in all of the 5 cases (21) (Fig. 1).

Analysis of $\mathrm{LOH}$ using microsatellite markers. We reconfirmed the existence of $\mathrm{LOH}$ in the specimens from the 30 cases using 3 microsatellite markers (D1S1189, D1S2151, and D1S2595) (Table II). A high frequency of LOH was found at the D1S1189 locus in 18/30 (60\%), D1S2151 locus in $16 / 30$ (53\%) and D1S2595 locus in 21/30 (70\%) (Fig. 2).

mRNA expression analysis. The expression levels of FAM5C mRNA were examined in 5 tongue SCC-derived cell lines, and two NOKs. All tongue SCC-derived cell lines exhibited decreased FAM5C mRNA expression compared with the NOKs. There was statistically significant difference in the

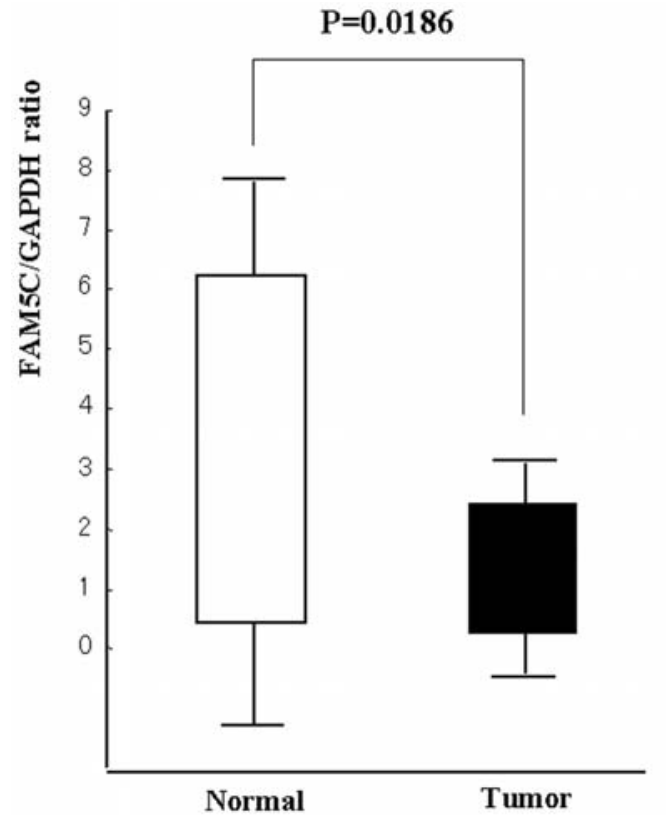

Figure 5. FAM5C mRNA expression status in primary tongue SCCs. The mRNA expression level of FAM5C was significantly reduced in tumors $(n=15)$ compared with the normal tongue tissues $(n=15)$. There was statistically significant difference in the FAM5C mRNA expression levels between tongue SCC and normal tissues ( $\mathrm{P}=0.0186$, Mann-Whitney $\mathrm{U}$ test).

FAM5C mRNA expression levels between tongue SCCderived cell lines and the NOKs $(\mathrm{P}=0.0472$, Mann-Whitney U test) (Fig. 3).

The expression levels of FAM5C mRNA were examined in the tumors, and paired normal oral tissues from the 15 of the specimens from the cases with tongue SCC. The relative mRNA expression levels in these primary tongue SCCs and the normal tissues ranged from 0.3 to 30.6 (mean: 1.44) and 0.04-37.4 (mean: 2.56), respectively. A significant decrease in FAM5C gene expression level was observed in $12(80 \%)$ of the 15 patient specimens (Fig. 4). The expression of FAM5C mRNA was significantly reduced in the tumors $(\mathrm{n}=15)$ compared with the normal tongue tissues $(n=15)$. There was statistically significant difference in the FAM5C mRNA expression levels between tongue SCCs and normal tissues ( $\mathrm{P}=0.0186$, Mann-Whitney $\mathrm{U}$ test) (Fig. 5). The data are

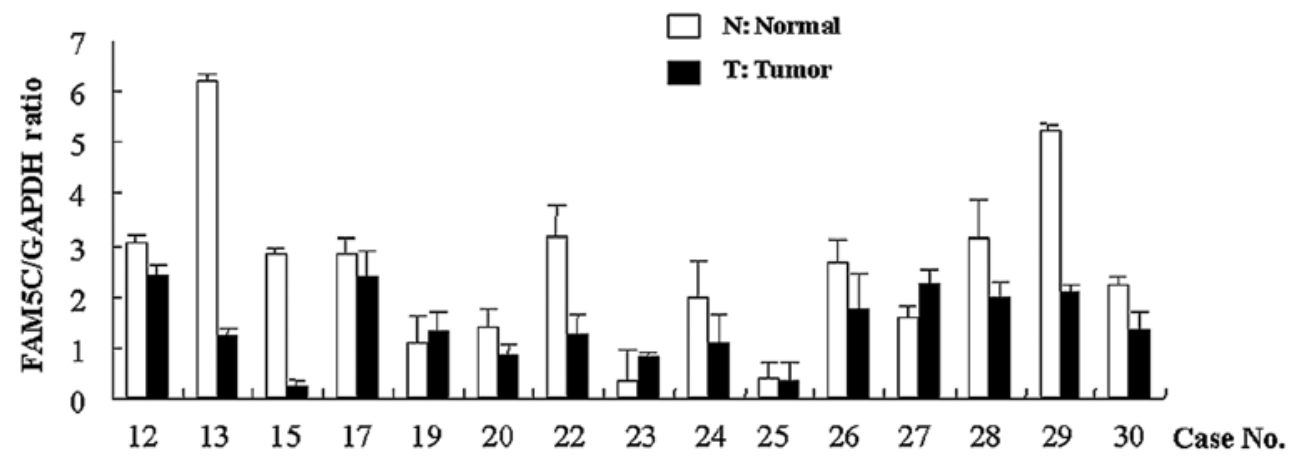

Figure 4. FAM5C mRNA expression status in primary tongue SCCs. The relative mRNA expression levels in the primary tongue SCCs and normal tissues ranged from 0.3 to 30.6 (mean: 1.44 ) and 0.04 to 37.4 (mean: 2.56), respectively. A significant decrease in $F A M 5 C$ gene expression was observed in 12 out of 15 patients $(80 \%)$. 
expressed as the mean \pm SD of three independent experiments with samples in triplicate.

Clinicopathological findings and statistical analysis. We compared our results with the clinicopathologic findings for each tumor. A significant statistical correlation between the incidence of LOH in the 1q31.1 region and TNM clinical stage status was observed. A number of the occurrences of $\mathrm{LOH}$ in the 1q31.1 region could be detected in early stage lesions; and the frequency of $\mathrm{LOH}$ tended to be higher in later clinical stages; however, there was no statistically significant correlation. Moreover, there was no statistically significant correlation between $\mathrm{LOH}$ and other clinicopathological findings such as pathological node, grade of histological differentiation, and prognosis associated with the tumor samples.

\section{Discussion}

Several studies have successfully utilized the Affymetrix $10 \mathrm{~K}$ SNP Mapping Array to identify consistent LOH regions $(1,3,4-15)$. However, there are no previous studies identifying CNA and $\mathrm{LOH}$ in the 1q31.1 region which we observed in the present study.

Allelic imbalances in whole-genome analyses of tongue SCCs have been reported using the Affymetrix 10K SNP Mapping Array (19). In that study, Zhou et al detected genome-wide LOH using the Affymetrix $10 \mathrm{~K}$ and $100 \mathrm{~K}$ SNP Mapping Array in tongue SCCs. The results from these two array platforms agreed closely, although more precise allelic imbalance patterns were revealed from the 100K SNP Mapping Array data. This suggests the feasibility of using the Affymetrix 10K SNP Mapping Array for genome-wide LOH detection in tongue SCCs. In addition, Zhou et al identified loss of CNA in the 3p11-p12 region. In our study, a rare loss of CNA (one copy) was observed in the region. We clearly established the copy number as one (from 0.8 to 1.2 ) by analysis of the DNA mapping array. This may be the reason that loss of CNA (one copy) was not observed frequently in the 3 p11-p12 region in our study.

We were able to observe a high frequency of $\mathrm{LOH}$ in the $1 \mathrm{q} 31.1$ region. The only gene known to exist in the $1 \mathrm{q} 31.1$ region is FAM5C. All tongue SCC-derived cell lines decreased FAM5C mRNA expression compared with the NOKs. There was statistically significant difference in the FAM5C mRNA expression levels between tongue SCC and normal tissues. These results further support the findings of our previous $\mathrm{LOH}$ analysis that the expression of FAM5C mRNA is decreased in tongue SCC.

Regarding the role of the FAM 5 C gene, it has been reported that the gene was correlated with the risk of myocardial infraction (MI) (22). To begin to address the functional role of FAM5C in MI, Connelly et al observed that FAM5C was expressed in the human aorta and that its transcript levels decreased with increasing passage of aortic smooth muscle cells in culture, suggesting that the level of gene expression may play a role in proliferation and senescence of this cell type. The correlations between MI and tongue SCC were not clear.

$F A M 5 C$ was originally identified in the mouse brain as a gene that is induced by the bone morphogenic protein and retinoic acid signaling (BRINP3) (23). BRINP3 is a novel protein of unknown function that is normally restricted to the brain. Shorts-Cary et al have shown that BRINP3 is a mitochondrially localized protein that is selectively upregulated in human gonadotropinomas (24). BRINP3 is overexpressed in pituitary gonadotrope cells and promotes proliferation, migration, and invasion, which suggests that it may play an important role in pituitary tumorigenesis. These findings may be useful for the functional analysis of the FAM5C gene.

The FAM5C gene is also designated deleted in bladder cancer chromosome region candidate 1-like (DBCCRl-like). The $D B C C R 1$ gene has been reported as the gene functionally affected by frequent loss of 9q32-33 in transitional cell carcinomas of the urinary bladder and identified as a candidate tumor suppressor, which is frequently targeted by promoter hypermethylation in bladder cancer (25). It has been reported that $\mathrm{LOH}$ at $9 \mathrm{q} 33$ and hypermethylation of the DBCCRI promoter are frequent and possibly early events in oral SCC development (26), however, Gao et al did not perform DNA mapping array analysis. In our whole-genome analysis of LOH, loss of CNA (one copy) was observed in the $9 q 33$ region. This suggests the $D B C C R 1$ gene may be a TSG of oral SCC.

Our results suggest that the $F A M 5 C$ gene may be a novel TSG peculiar to tongue SCC, and inactivation of the FAM5C gene may play one or more roles in the carcinogenesis of tongue SCCs. In the present study, we established that analysis of DNA mapping array using the Affymetrix 10K SNP Mapping Array was an efficient method to detect genomewide cancer-associated LOH for tongue SCC. A significant statistical correlation between the incidence of genome-wide cancer-associated LOH and TNM clinical stage status were not clear. We were not able to apply the functional analysis of FAM5C gene. Further studies such as immunoblotting, immunofluorescence, and immunohistochemistry are needed to clarify its function in the development and progression of tongue SCC. Furthermore, increasing the number of patient specimens may make it possible to detect genome-wide cancer-associated LOH for tongue SCC in regions other than the $1 \mathrm{q} 31.1$ region.

\section{Acknowledgements}

This work was supported by a Research Grant from the Ministry of Education, Science and Culture, Japan (No. 20791549).

\section{References}

1. Janne PA, Li C, Zhao X, et al: High-resolution singlenucleotide polymorphism array and clustering analysis of loss of heterozygosity in human lung cancer cell lines. Oncogene 23: 2716-2726, 2004.

2. Yamamoto N, Mizoe J, Numasawa H, Tsujii H, Shibahara T and Noma H: Allelic loss on chromosomes 2q, 3p and 21q: possibly a poor prognostic factor in oral squamous cell carcinoma. Oral Oncol 39: 796-805, 2003.

3. Zhou X, Li C, Mok SC, Chen Z and Wong DT: Whole genome loss of heterozygosity profiling on oral squamous cell carcinoma by high-density single nucleotide polymorphic allele (SNP) array. Cancer Genet Cytogenet 151: 82-84, 2004.

4. Huang J, Wei W, Zhang J, et al: Whole genome DNA copy number changes identified by high density oligonucleotide arrays. Hum Genomics 1: 287-299, 2004. 
5. Zhao X, Li C, Paez JG, et al: An integrated view of copy number and allelic alterations in the cancer genome using single nucleotide polymorphism arrays. Cancer Res 64: 3060-3071, 2004.

6. Schubert EL, Hsu L, Cousens LA, et al: Single nucleotide polymorphism array analysis of flow-sorted epithelial cells from frozen versus fixed tissues for whole genome analysis of allelic loss in breast cancer. Am J Pathol 160: 73-79, 2002.

7. Wang ZC, Lin M, Wei LJ, et al: Loss of heterozygosity and its correlation with expression profiles in subclasses of invasive breast cancers. Cancer Res 64: 64-71, 2004.

8. Paez JG, Lin M, Beroukhim R, et al: Genome coverage and sequence fidelity of phi29 polymerase-based multiple strand displacement whole genome amplification. Nucleic Acids Res 32: E71, 2004

9. Primdahl H, Wikman FP, von der Maase $\mathrm{H}$, Zhou XG, Wolf $\mathrm{H}$ and Orntoft TF: Allelic imbalances in human bladder cancer: genome-wide detection with high-density single-nucleotide polymorphism arrays. J Natl Cancer Inst 94: 216-223, 2002.

10. Hoque MO, Lee CC, Cairns P, Schoenberg M and Sidransky D Genome-wide genetic characterization of bladder cancer: a comparison of high-density single-nucleotide polymorphism arrays and PCR-based microsatellite analysis. Cancer Res 63: 2216-2222, 2003.

11. Lieberfarb ME, Lin M, Lechpammer M, et al: Genome-wide loss of heterozygosity analysis from laser capture microdissected prostate cancer using single nucleotide polymorphic allele (SNP) arrays and a novel bioinformatics platform dChipSNP. Cancer Res 63: 4781-4785, 2003.

12. Dumur CI, Dechsukhum C, Ware JL, et al: Genome-wide detection of $\mathrm{LOH}$ in prostate cancer using human SNP microarray technology. Genomics 81: 260-269, 2003.

13. Wong KK, Tsang YT, Shen J, Cheng RS, Chang YM, Man TK and Lau CL: Allelic imbalance analysis by high-density singlenucleotide polymorphic allele (SNP) array with whole genome amplified DNA. Nucleic Acids Res 32: E69, 2004.

14. Lindblad-Toh K, Tanenbaum DM, Daly MJ, et al: Loss-ofheterozygosity analysis of small-cell lung carcinomas using single-nucleotide polymorphism arrays. Nat Biotechnol 18 : 1001-1005, 2000.

15. Zhou X, Mok SC, Chen Z, Li Y and Wong DT: Concurrent analysis of loss of heterozygosity $(\mathrm{LOH})$ and copy number abnormality (CNA) for oral premalignancy progression using the Affymetrix $10 \mathrm{~K}$ SNP mapping array. Hum Genet 115 $327-330,2004$
16. Onda T, Uzawa K, Endo Y, et al: Ubiquitous mitochondrial creatine kinase downregulated in oral squamous cell carcinoma. Br J Cancer 94: 698-709, 2006.

17. Kato H, Uzawa K, Onda T, et al: Down-regulation of 1D-myoinositol 1,4,5-trisphosphate 3-kinase A protein expression in oral squamous cell carcinoma. Int J Oncol 28: 873-881, 2006.

18. Suzuki N, Onda T, Yamamoto N, Katakura A, Mizoe JE and Shibahara T: Mutation of the p16/CDKN2 gene and loss of heterozygosity in malignant mucosal melanoma and adenoid cystic carcinoma of the head and neck. Int J Oncol 31: 1061-1067, 2007.

19. Zhou X, Teman S, Chen Z, Ye H, Mao L and Wong DT: Allelic imbalance analysis of oral tongue squamous cell carcinoma by high-density single nucleotide polymorphism arrays using whole-genome amplified DNA. Hum Genet 118: 504-507, 2005.

20. Endo Y, Uzawa K, Mochida Y, et al: Sarcoendoplasmic reticulum $\mathrm{Ca}(2+)$ ATPase type 2 down-regulated in human oral squamous cell carcinoma. Int J Cancer 110: 225-231, 2004.

21. Kuroiwa T, Yamamoto N, Onda T, et al: Analysis of copy number abnormality (CNA) and loss of heterozygosity (LOH) on whole genome using single nucleotide polymorphism (SNP) genotyping arrays in tongue squamous cell carcinoma. Jpn J Oral Maxillofac Surg 54: 316-322, 2008.

22. Connelly JJ, Shah SH, Doss JF, et al: Genetic and functional association of FAM5C with myocardial infarction. BMC Med Genet 9: 33, 2008.

23. Kawano H, Nakatani $\mathrm{T}$, Mori $\mathrm{T}$, et al: Identification and characterization of novel developmentally regulated neuralspecific proteins, BRINP family. Brain Res Mol Brain Res 125: 60-75, 2004.

24. Shorts-Cary L, Xu M, Ertel J, et al: Bone morphogenetic protein and retinoic acid-inducible neural specific protein-3 is expressed in gonadotrope cell pituitary adenomas and induces proliferation, migration, and invasion. Endocrinology 148: 967975, 2008

25. Beetz C, Brodoehl S, Patt S, Kalff R and Deufel T: Low expression but infrequent genomic loss of the putative tumor suppressor DBCCRI astrocytoma. Oncol Rep 13: 335-340, 2005.

26. Gao S, Worm J, Guldberg P, et al: Loss of heterozygosity at $9 \mathrm{q} 33$ and hypermethylation of the $D B C C R 1$ gene in oral squamous cell carcinoma. Br J Cancer 91: 760-764, 2004. 